\title{
Headache at stroke onset: the Lausanne Stroke Registry
}

\author{
Emre Kumral, Julien Bogousslavsky, Guy Van Melle, Franco Regli, Philippe Pierre
}

\begin{abstract}
Within 12 hours of stroke onset 2506 patients with first ever stroke admitted to the Lausanne Stroke Registry were questioned about headache. Eighteen per cent of the patients reported headache, $14 \%$ with anterior circulation stroke and $29 \%$ with posterior circulation stroke $(\mathrm{p}<0.001)$. Headache was reported by $16 \%$ of the patients with infarct and $36 \%$ of those with haemorrhage $(p<0.001)$. The prevalence of headache was $9 \%$ with lacunar infarct, $15 \%$ with middle cerebral artery territory infarct, $37 \%$ with infratentorial haemorrhage, and $36 \%$ with supratentorial haemorrhage. The most common topography of pain was frontal (41\%), followed by diffuse headache $(27 \% ; p<0.001)$. Diffuse $(41 \%)$ or occipital $(30 \%)$ headache was particularly frequent with posterior circulation stroke, whereas frontal headache was associated with anterior circulation stroke (51\%; $p<0.001)$. Headache in stroke may be explained in part by involvement of blood vessels (acute distention or distortion) and mechanical (stretch of haemorrhage) stimulation of intracranial nociceptive afferents. Stroke due to dissection was strongly associated with headache $(p<0.001)$, whereas embolic (cardiac, artery to artery) stroke was more common without headache ( $p$ $<0.001$ ), emphasising the role of extracranial $v$ intracranial arteries in the genesis of headache at stroke onset. Moreover, dual trigeminal-vascular and cervical-vascular system involvement in causing headache may explain the lack of correspondence with the "rules of referral" in up to $38 \%$ of the cases.
\end{abstract}

(F Neurol Neurosurg Psychiatry 1995;58:490-492)

Keywords: headache; stroke; brain haemorrhage; cerebral infarct

Headache is commonly reported in stroke, but its frequency varies from study to study. ${ }^{1-8}$ The occurrence of headache is different between patients with large artery disease and those with small artery disease..$^{2-4} 7$ Also, there may be a relation between the localisation of stroke and the type of headache, ${ }^{17-10}$ but there is some disagreement about the reliability of the correlations. We have studied headache at stroke onset in patients consecutively included in the Lausanne Stroke Registry. ${ }^{11}$ We assessed the relation between headache, stroke localisation, aetiology, history, and clinical findings. The findings were compared with those in patients without headache admitted during the same period.

\section{Patients and methods}

For years we investigated 2506 patients who had had an initial stroke, with a standard protocol including blood and urine tests, brain CT (at least once between day 3 and day 7 after the stroke), Doppler ultrasound, 12 lead ECG, brain MRI, two dimensional echocardiography, and cerebral angiography in selected cases. All patients had CT (one to five examinations) or MRI with or without contrast and all were included in the Lausanne Stroke Registry. ${ }^{11}$ This is a prospective registry of first ever stroke patients admitted to a primary care centre. In cerebral infarcts, the vascular territory involved was assessed with templates developed in our centre and elsewhere. ${ }^{12} \mathrm{We}$ classified infarcts in the anterior circulation into infarcts in the middle cerebral artery (MCA) territory), border zone infarcts, multiple infarcts, and infarcts in the anterior cerebral artery (ACA) territory. Posterior circulation infarcts were classified according to the anatomical and vascular locations.

We considered the following main aetiologies for brain infarct: (a) large artery disease, defined as narrowing of $>50 \%$ of the lumen diameter of the large artery appropriate to the infarct; (b) potential cardiac sources of embolism, defined as intracardiac thrombus or tumour, mitral stenosis, prosthetic aortic or mitral valves, endocarditis, left ventricular aneurysm or akinesia, paradoxical embolism associated with patent foramen ovale, mitral valve prolapse, interatrial septum aneurysm, atrial fibrillation, or sick sinus syndrome; (c) lacunar infarction, defined as a small $(<1.5 \mathrm{~cm})$ deep infarct in a patient with known hypertension or diabetes mellitus, in the absence of another cause of stroke; (d) other and undetermined. In cerebral 
haemorrhage, we considered five main localisations: lenticular-capsular, lobar (frontal, temporal, parieto-occipital), cerebellar, thalamic, and brainstem.

Headache at the onset of stroke was defined as cephalic pain appearing just before or after other stroke symptoms. We assessed headache in all patients with a routine neurological questionnaire. Relatives were asked about the history of headache at the onset of stroke if the patients were unconscious, confused, or aphasic. Every patient's questionnaire was completed and we did not consider the patients who could not give any information. We investigated systematically the localisation of headache (diffuse, frontal, lateral, occipital, and fronto-occipital). Risk factors were defined according to Registry guidelines. ${ }^{11}$ To detect potential differences between patients with headache and those without we compared risk factors, clinical state, and type, causes, and localisation of stroke in these two groups. Both groups underwent the same protocol of investigations within the frame of the Registry. Statistical analysis was by $\chi^{2}$ test, Fisher's exact test, and analysis of variance (ANOVA).

Table 1 Stroke type and localisation

\begin{tabular}{lcc}
\hline & $\begin{array}{c}\text { Headache group } \\
(n=456)\end{array}$ & $\begin{array}{c}\text { Non-headache group } \\
(n=2050)\end{array}$ \\
\hline Ischaemic stroke & 365 & 1980 \\
Anterior circulation infarct & 176 & 1253 \\
MCA infarct & $43(9)$ & $176(9)$ \\
MCA superficial anterior infarct & $44(10)$ & $316(16)$ \\
MCA superficial posterior infarct & $32(7)$ & $222(11)$ \\
ACA infarct & $5(1)$ & $38(2)$ \\
MCA + MCA infarct & $7(2)$ & $10(0 \cdot 1)$ \\
Subcortical infarct & $45(9)$ & $481(23)$ \\
Posterior circulation infarct & 149 & 401 \\
PCA superficial infarct & $36(8)$ & $70(3)$ \\
PCA superficial + deep & $11(2)$ & $14(1)$ \\
Thalamic infarct & $14(3)$ & $69(3)$ \\
Brainstem infarct & $61(13)$ & $201(10)$ \\
Cerebellum & $27(6)$ & $47(2)$ \\
Multiple infarcts & $32(7)$ & $197(9)$ \\
Border zone infarct & $8(2)$ & $39(2)$ \\
Haemorrhagic stroke & 91 & \\
Striatocapsular haemorrhage & $27(6)$ & 760 \\
Parietooccipital haemorrhage & $23(5)$ & $26(3)$ \\
Frontal haemorrhage & $16(3)$ & $21(1)$ \\
Temporal haemorrhage & $9(2)$ & $15(1)$ \\
Thalamic haemorrhage & $5(1)$ & $9(0 \cdot 1)$ \\
Brainstem haemorrhage & $7(2)$ & $9(0 \cdot 1)$ \\
Cerebellar haemorrhage & $4(0 \cdot 8)$ & $9(0 \cdot 1)$ \\
\hline ACA Anterior cerebral arter & &
\end{tabular}

ACA = Anterior cerebral artery; $\mathrm{MCA}=$ middle cerebral artery; $\mathrm{PCA}=$ posterior cerebra artery. Results are numbers of patients (\%).

Table 2 Location of headache and stroke topography

\begin{tabular}{lcllll}
\hline & \multicolumn{2}{l}{ Ischaemic stroke } & & \multicolumn{2}{l}{ Haemorrhagic stroke } \\
\cline { 2 - 3 } \cline { 5 - 6 } & $\begin{array}{l}\text { Anterior } \\
\text { circulation } \\
(n=200)\end{array}$ & $\begin{array}{l}\text { Posterior } \\
\text { circulation } \\
(n=165)\end{array}$ & & $\begin{array}{l}\text { Supratentorial } \\
(n=52)\end{array}$ & $\begin{array}{l}\text { Infratentorial } \\
(n=39)\end{array}$ \\
\hline Diffuse & $51(26)$ & $34(21)$ & & $20(38)$ & $16(41)$ \\
Frontal & $102(51)$ & $56(34)$ & & $20(38)$ & $9(23)$ \\
Lateral & $20(10)$ & $15(20)$ & & $6(12)$ & $5(13)$ \\
Occipital & $21(11)$ & $49(30)$ & & $1(2)$ & $6(15)$ \\
Fronto-occipital & $6(3)$ & $11(2)$ & & $5(10)$ & $3(8)$ \\
\hline
\end{tabular}

Values are numbers of patients (\%). Comparison of four types of stroke (anterior and posterio ischaemic stroke, supratentorial and infratentorial haemorrhagic stroke) yields $\chi^{2} 209 \cdot 30$; $p<$ 0.001 . The difference is mainly due to the high prevalence of frontal and diffuse headache in anterior circulation stroke and of occipital and occipitofrontal headache in posterior circulation stroke.

\section{Results}

Eighteen per cent of our patients with initial stroke presented with headache at the onset of stroke. There were 456 patients with headache (204 women, 252 men, mean age $54 \cdot 2$ (SD 16.5), range 17-91 years) and 2050 patients without headache (740 women, 1310 men, mean age 64.5 (SD 13.2) range 16-94 years; $\mathrm{p}<0.001)$. Headache was reported by $16 \%(365 / 2255)$ of the patients with infarct and by $36 \%(91 / 251)$ of the patients with haemorrhage $(p<0.001)$. Headache was present in $14 \%(252 / 1810)$ of the patients with anterior circulation stroke and in $29 \%$ $(204 / 696)$ of the patients with posterior circulation stroke $(\mathrm{p}<0.001)$.

Diabetes (48\%), smoking (33\%), cardiac ischaemia $(24 \%)$, and transient ischaemic attacks $(23 \%)$ were significantly more frequent in the patients without headache. The most remarkable factor associated with headache was a history of migraine ( $p<$ $0.001)$. Large artery disease, lacunar infarct, and potential cardiac sources of emboli were more frequent without than with headache $(p<0.001)$. Undetermined and rare causes (including arteritis, fibromuscular dysplasia, cerebral venous thrombosis, and coagulopathy) were more common with headache (14\% and $16 \% v 11 \%$ and $4 \%$; ( $<<0.001)$. Dissections (extracranial internal carotid artery or vertebral artery) showed the strongest association with headache $(p<0.001)$.

\section{NEUROLOGICAL FINDINGS}

The type of stroke onset was significantly different $(p<0.001)$ between the two groups. The stroke was stabilised within less than one hour in $53 \%$ of the patients with headache $v 74 \%$ in the patients without headache; on the other hand, it was progressive over more than one hour in $24 \%$ of the patients $v 13 \%$ of the patients without headache. Aphasia was more frequent (45\% $v \quad 30 \%$; $<0.001$ ) without headache. Somnolence at stroke onset was significantly more frequent with than without headache $(22 \% v 10 \% ; \mathrm{p}<0.001)$. Death rate at one month was identical with or without headache. Motor weakness was significantly more common without than with headache $(86 \% v 22 \% \mathrm{p}<0.001)$, contrary to sensory deficits, which were more often encountered with headache $(p<0.01)$.

\section{LESION TYPE AND LOCALISATION (TABLE 1)}

The patients with posterior circulation ischaemia more often had headache $(27 \%)$ than the patients with anterior circulation ischaemia $(12 \%)(p<0.001)$. Headache was found in $9 \%(61 / 697)$ of the patients with lacunar infarct, and in $15 \%(126 / 860)$ of those with a middle cerebral artery infarct ( $p$ $<0.01$ ). The frequency of headache in posterior circulation infarct was $27 \%(165 / 604)$. Headache was present in $37 \%$ of the patients with infratentorial haemorrhage (16/43) and in $36 \%$ of the patients with supratentorial haemorrhage $(75 / 208)$. 
TYPE OF HEADACHE (TABLE 2)

Among 456 patients with headache, the localisation was frontal $(41 \%)$, diffuse $(27 \%)$, occipital $(17 \%)$, lateral $(10 \%)$, and frontooccipital (5\%). In ischaemic stroke, frontal headache was present in $51 \%$ of the patients with anterior circulation infarct, whereas occipital headache was generally seen with posterior circulation infarct $(30 \%) \quad(p<$ $0.001)$; $168 / 456$ (38\%) patients had headache localised to another region than the location of stroke, including $14 \%(27 / 200)$ of the cases with anterior circulation infarct, $64 \%(105 / 165)$ of the cases with posterior circulation infarct, $12 \%(6 / 52)$ of the cases with supratentorial haemorrhage, and $77 \%$ (30/39) of the cases with infratentorial haemorrhage.

Five of 13 patients with arteritis had frontal headache. Five of 14 patients with coagulopathy and cerebral haemorrhage had diffuse headache. All 10 patients with cerebral venous thrombosis had diffuse headache.

\section{Discussion}

In our study, the frequency of headache at onset of stroke was $18 \%$, which lies close to the range of other studies $(5-17 \%) .{ }^{34-9} \mathrm{We}$ found that headache was more common in men and younger patients, but other studies reported a higher prevalence in women. ${ }^{4713}$

Headache may occur more often in haemorrhagic than in ischaemic stroke. We also noted a high rate of headache in posterior circulation stroke; this agrees with previous studies, which reported a frequency of 25-54\%. ${ }^{1-1013}$ Headache was not common in lacunar infarcts, as already reported in other studies, where it ranged between $4 \%$ and $17 \% .^{7-9}$

Haemorrhagic stroke was the most common cause of headache in our series. Other authors reported a frequency of headache of $23-68 \%$ with ganglionic or lobar haemorrhage. ${ }^{257814}$ In our study haemorrhagic strokes generally led to diffuse headache (mainly with infratentorial haemorrhage). It is probable that in haemorrhage, diffuse headache is caused by mechanical stretch and by blood products that diffuse and irritate the trigeminovascular system at the basis of the skull.

In our study the most relevant cause of headache was arterial dissection. Perhaps dissection may lead to pain by stretching and distorting the involved extracranial vessel, causing a mechanical stimulus to the trigeminovascular system. It is interesting that our findings suggest that acute dilatation or stretch of intracranial arteries by atheromatous or cardiac emboli may not be as important in producing pain as previously claimed. ${ }^{10} 15$

In general, the location of headache correlated well with the localisation of stroke. ${ }^{2-10}$ Frontal headache predominated in anterior circulation infarct and occipital headache was mostly found with posterior circulation infarct. This topological correlation could be explained by a physical or chemical stimulation of the trigeminovascular system in the affected brain region. The headache was referred to another region, however, in over one third of the cases.

In our present study, the patients with headache more often had a history of migraine. It is possible that migraineurs may be predisposed to develop headache at stroke onset more often than non-migraineurs. Also, pain in these two situations may have a common mechanism. Edmeads ${ }^{13}$ postulated an important role for platelet adhesion, aggregation, and release in headache of atherothrombotic stroke, processes that might also be involved in the genesis of migraine headache.

The mechanism of headache at stroke onset is not clear, but most studies agree that headache may be more frequent in posterior circulation stroke and in dissections. The explanation could be that vessels in the posterior circulation are more richly innervated by nociceptive afferents than in the anterior circulation. ${ }^{816}$ Headache is probably induced through a stimulation of the trigeminal-vascular system, ${ }^{17}$ and its localisation frequently corresponds to the localisation of the brain lesion. On the other hand, the dual trigeminal and cervical innervation of the cerebral vascular system involved in causing headache may explain the lack of correspondence with the "rules of referral" in many cases.

1 Edmeads J. The headaches of ischaemic cerebrovascular disease. Headache 1979;19:345-9.

Fisher CM. Headache in cerebrovascular disease. In Vinken PJ, Bruyn GW, eds. Handbook of clinical neurology. Vol 5. Amsterdam: North Holland Publishing Co 1968:124-56.

3 Gorelick P, Caplan LR, Langenberg P. Headache in acute cerebrovascular disease. Neurology 1986;36:1445-50.

4 Grindal AB, Toole JF. Headache and transient ischemic attacks. Stroke 1974;5:603-6.

5 Mohr JP, Caplan LR, Melski JW. The Harvard Cooperative Stroke Registry: a prospective registry. Neurology 1978;28:754-62.

6 Loeb C, Gandolfo C. Headache in ischemic cerebrovascular disease. Neurology 1987;37:1266.

7 Portenoy RK, Abissi CJ, Lipton RB, Berger AR, Mebler MF, Baglivo J, et al. Headache in cerebrovascular disease. Stroke 1984;15:1009-12.

8 Vestergaard $\mathrm{K}$, Andersen G, Nielsen MI, Jensen TS Headache in stroke. Stroke 1993;24:1621-4.

9 Koudstaal PJ, van Gijn J, Kappelle J, for the Dutch TIA Study Group. Headache in transient or permanent cerebral ischemia. Stroke 1991;22:754-9.

10 Auerbach SH, Butler RB, Levine HL. Headache in cerebral embolic disease. Stroke 1981;12:367-9.

11 Bogousslavsky J, Van Melle G, Regli F. The Lausann Stroke Registry. Analysis of 1000 consecutive patients with first stroke. Stroke 1988;19:1083-92.

12 Bogousslavsky J, Nater B, Vingerhoets FG. Sémiologie des AVC ischémiques. Syndromes artériels ischémiques cérébraux. In: Bogousslavsky J, Bousser MG, Mas JL eds. Accidents vasculaires cérébraux. Paris: Doin Editeurs, 1993:110-37.

13 Edmeads J. Complicated migraine and headache in cerebrovascular disease. Neurol Clin 1983;1:385-97.

14 Airing CD, Merritt HH. Differential diagnosis between cerebral hemorrhage and cerebral thrombosis: a clinical and pathologic study of 245 cases. Arch Intern Med and pathologic

15 Dalessio DJ. Wolff's headache and other pain. New York: Oxford University Press, 1972:189-212.

16 Olesen J. Clinical and pathophysiological observations in migraine and tension-type headache explained by vascular, supraspinal and myofascial inputs. Pain 1991;46: 125-32.

17 Moskowitz MA. Neurobiology of vascular head pain. Ann Neurol 1984;16:157-62. 